\title{
The remarkable difference between surface and step atoms in the magnetic anisotropy of two-dimensional nanostructures
}

\author{
S. RUSPONI, T. CREN, N. WEISS, M. EPPLE, P. BULUSCHEK, L. CLAUDE AND H. BRUNE* \\ Institute of the Physics of Nanostructures, Ecole Polytechnique Fédérale de Lausanne (EPFL), CH-1015 Lausanne, Switzerland \\ *e-mail: harald.brune@epfl.ch
}

Published online: 27 J uly 2003; doi:10.1038/nmat930

The original magnetic properties of nanometre-sized particles are due to the distinct contributions of volume, surface and step atoms. To disentangle these contributions is an ongoing challenge of materials science. Here we introduce a method enabling the identification of the remarkably different contributions of surface and perimeter atoms to the magnetic anisotropy energy of twodimensional nanostructures. Our method uses the generally nonlinear relationship between perimeter length and surface area. Atomic-scale characterization of the morphology of ensembles of polydisperse nanostructures, combined with in situ measurements of their temperature-dependent magnetic susceptibility, gives access to the role played by the differently coordinated atoms. We show for Co nanostructures on a Pt(111) surface that their uniaxial out-ofplane magnetization is entirely caused by edge atoms having 20 times more anisotropy energy than their bulk and surface counterparts. Identification of the role of perimeter and surface atoms opens up unprecedented opportunities for materials engineering. As an example, we separately tune magnetic hardness and moment in bimetallic core-shell nanostructures. b xploring the ultimate density limits of magnetic information storage, whether on computer hard disks or in MRAMs (magnetic random access memories), requires elaborate tuning of the preferred (easy) magnetization axis, of the magnetic anisotropy energy, and of the magnetic moment in the units used to store a bit. These units are single-domain particles (with diameter $d<20 \mathrm{~nm}$ ) where the magnetic moments of all atoms are ferromagnetically aligned $^{1}$ to form the overall magnetic moment of the particle $M$, which is also called the macrospin. The preferred orientations of $M$, and the anisotropy energy barriers $K$ separating them, are given by the delicate balance between several competing energies. These are the magnetocrystalline bulk anisotropy, its surface and step counterparts, and the shape anisotropy, or demagnetizing energy, resulting from the interaction of $M$ with its own dipolar stray field. Unravelling the anisotropy's origin is far from trivial due to the competition between these energies ${ }^{2}$. This is unfortunate because the anisotropy is one of the key quantities: it defines the stability of the magnetization direction against thermal excitation, and therefore the minimum particle size for which non-volatile information storage may be achieved (at $300 \mathrm{~K}$ this requires $K \geq 1.2 \mathrm{eV}$ ). A further key parameter is the modulus of $M, M$ defining the dipolar stray field used to read and write, but also mediating interactions between adjacent bits. These interactions are minimized for out-of-plane magnetization, and because the ultimate limit of single-particle bits may only be achieved for uniaxial systems, uniaxial out-of-plane systems are best suited to explore the ultimate density limit of magnetic recording ${ }^{3,4}$.

Current studies attempting to identify the origin of magnetic anisotropy mainly deal with two model systems. These are colloids or three-dimensional (3D) nanoparticles, and 2D nanostructures created by molecular-beam epitaxy at single-crystal surfaces. For colloid particles, remarkable progress has been achieved in monodispersity ${ }^{5}$, their self-assembly into $2 \mathrm{D}$ superlattices ${ }^{6,7}$ and in the accomplished anisotropy energies per constituent atom ${ }^{8}$. Despite their promising properties for applications, 3D nanoparticles present several difficulties for tracing back the origin of anisotropy. First, although the magnetism of a single particle can be addressed ${ }^{9}$, it is almost impossible to study the morphology of the very same particle in conjunction with its magnetism. Second, the particles frequently have a few atomic layers of oxide at their surface, which is not ferromagnetic ${ }^{10}$. This causes uncertainties in the morphology of the ferromagnetically ordered 


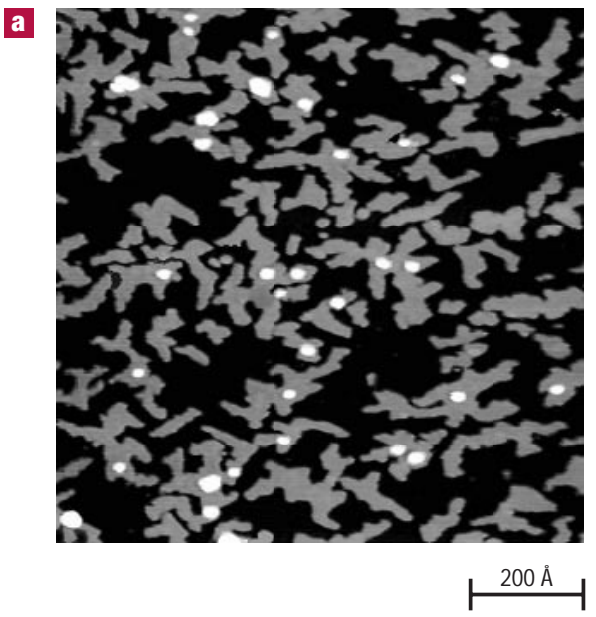

G

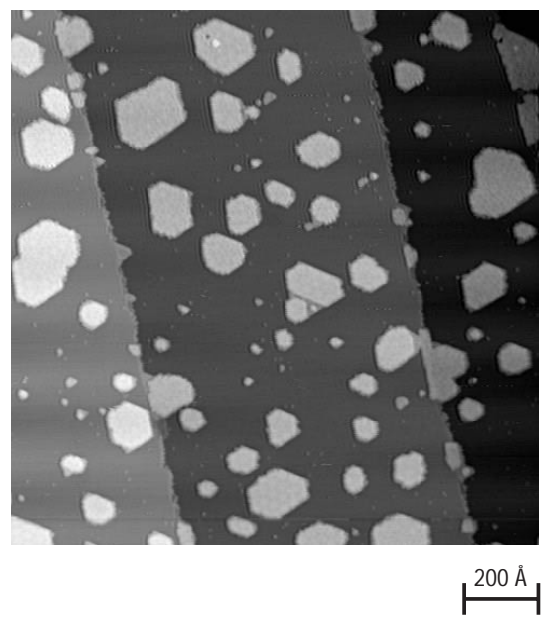

b.

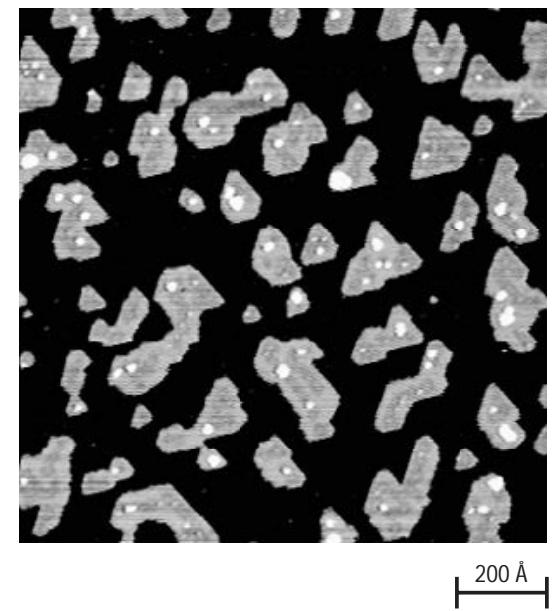

d

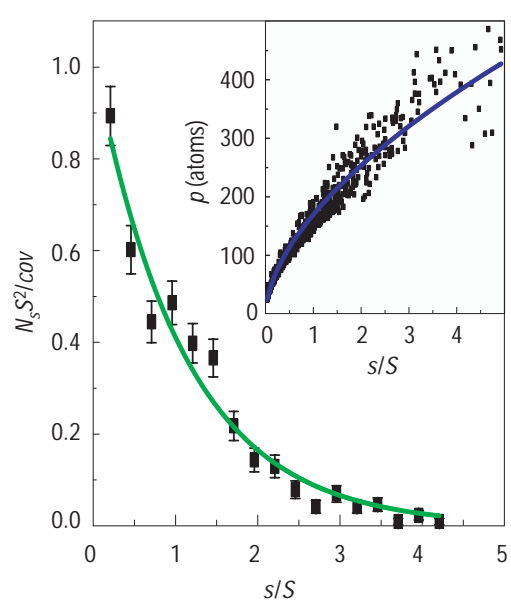

Figure 1 Co islands created on a Pt(111) surface. a- c, Scanning tunnelling microscope (STM) images showing three morphologies: a, Ramified islands, one atomic layer high (coverage 0.40 monolayers, one monolayer is defined as one $C 0$ atom per Pt substrate atom). $\mathbf{b}$, Compact one-monolayer-high islands. The bright patches in $\mathbf{a}$ and $\mathbf{b}$ are islands nucleated in the second layer. c, Double-layer islands. All islands a-c care pure Co as concluded from the constantAuger electron spectra Co/Pt peak-to-peak ratio on annealing, and from the absence of heterogeneity in the STM images, which clearly reveal $\mathrm{Co}$ and Pt as having different apparent heights (see Fig. 5). d, Size distribution of the islands shown in $\mathbf{b}$ obtained from a statistical ensemble of 1,000 islands. As customary in literature on epitaxial growth, this distribution has been normalized to yield unit area under the curve ( $s$ island size, $S$ average island size, $N_{s}$ density of islands of size $s$, cov coverage in monolayers). The inset shows the perimeter length $p$ as a function of island size. Solid lines are fits to these distributions used as input for calculating $\chi(T)$; using the individual data points produces identical results.

particle core. Owing to these difficulties, no general view on the origin of anisotropy in nanoparticles has yet evolved. The anisotropy has either

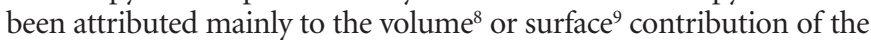
crystalline lattice, or to shape anisotropy caused by eccentricities of the magnetic core ${ }^{10}$. In addition to the difficult access of the anisotropy's origin, the desired uniaxial media have not yet been realized with 3D nanoparticles because an individual particle can have several easy magnetization axes. In addition, the deposited particles are randomly oriented, as are, therefore, their preferred axes ${ }^{11}$.

Two-dimensional nanostructures at surfaces are optimally suited to address the origin of anisotropy, and also to explore density limits of non-interacting magnetic bits. The anisotropy imposed by the substrate makes them uniaxial, for example oriented out-of-plane ${ }^{12}$. The size and spatial uniformity achieved by self-assembly techniques ${ }^{13,14}$ are comparable to those of colloids. Under ultrahigh vacuum conditions, there is no oxide shell and all constituent atoms are ferromagnetically ordered. Thus the particle morphology contributing to the magnetic signal is accessible at the atomic scale, and can be related to the magnetic properties measured in situ. Here we made use of this to determine the magnetic anisotropy energy of surface versus perimeter atoms. We used 2D islands with broad size and perimeter length distributions to probe large size and perimeter length intervals with a single sample. The nonlinear relationship between perimeter length and island area gives each of the two distributions its characteristic shape, enabling the disentanglement of the roles of the two atomic species. We performed in situ measurements of the temperature dependence of the magnetic susceptibility with magneto-optical Kerr effect (MOKE), and analyse the data with an analytical model describing the blocking to superparamagnetic transition of a monodomain particle. Using the scanning tunnelling microscope (STM)-derived area and perimeter distributions as input leaves the anisotropy per perimeter and surface atom as the only two fit parameters. The result is a surprisingly large difference between perimeter and surface atoms. The MOKE and STM equipment used were home-built. 
To study the role of perimeter versus surface atoms we consider the three island morphologies shown in Fig. 1. The islands have been created by Co evaporation from a carefully outgassed Co rod (purity 99.995\%, Alfa Aesar) onto a clean $\mathrm{Pt}(111)$ surface at a vacuum pressure below $8 \times 10^{-11}$ mbar using a commercial electron-beam evaporator (base pressure of the ultrahigh vacuum chamber $3 \times 10^{-11} \mathrm{mbar}$ ). The ramified islands in Fig. 1a form on deposition at $130 \mathrm{~K}$ due to limited mobility of atoms along the island edge ${ }^{15}$. Annealing them to $300 \mathrm{~K}$ creates edge mobility yielding compact islands, see Fig. 1b. Further annealing to $340 \mathrm{~K}$ converts the monolayer-high islands of Fig. 1b into double-layer islands represented in Fig. 1c. This mutation is caused by the surface and interface free energies favouring atoms climbing up onto the second layer and thereby reducing, with respect to $\mathrm{Pt}(111) /$ vacuum, the more costly $\mathrm{Co} /$ vacuum and $\mathrm{Co} / \mathrm{Pt}$ interfaces by a factor of two. For all three island morphologies shown in Fig. 1, we find a nonlinear relationship between perimeter length and area. For brevity we now focus on the case in Fig. 1b, for which the size and perimeter distributions are shown in Fig. 1d. The mean island size is $S=1,200 \pm 1,000$ atoms, and the perimeter length $p$ (in number of atoms) is related to the area $a$ by $p=\alpha a^{\beta}$, with $\alpha=2.8$ and $\beta=0.6$ in this specific case, this is equivalent to $a=0.17 p^{1.7}$ (for square islands $a=1 / 16 p^{2}$ and for circular ones $\left.a=1 / 4 \pi p^{2}\right)$.

Before analysing the experimental data of the temperaturedependent zero-field susceptibility $\chi(T)$ in terms of anisotropy energies of surface and step atoms, we present an analytical model describing the transition from blocking to superparamagnetism of an ensemble of monodisperse, non-interacting particles. The Co particles have uniaxial out-of-plane anisotropy and thus are characterized by two local minima for the potential energy $E$ as a function of the angle $\Theta$ between surface normal and magnetic moment $M$. These minima correspond to the up and down orientation of $M$ (see inset of Fig. 2). The anisotropy energy at zero field $(H=0)$ is the energy $K=E\left(90^{\circ}\right)$, associated with the in-plane configuration forming a transition state that has to be overcome during magnetization reversal. The rate of barrier crossing for an ensemble of monodisperse particles, all having identical values of $K$ and $M$, or the time average of this rate for a single particle, is described for our case of small fields by an Arrhenius expression $v=v_{0} \exp \left((-K \pm H M) / \mathrm{k}_{\mathrm{B}} T\right)$, with $\mathrm{k}_{\mathrm{B}}$ being the Boltzmann constant; typically $v_{0}=1 \times 10^{10} \mathrm{~s}^{-1}$ (Ref. 16). At zero field, the relaxation time $\tau$ is given by $\tau=1 / 2 v=\tau_{0} \exp \left(K / \mathrm{k}_{\mathrm{B}} T\right)$, with $\tau_{0}=1 / 2 v_{0}$. The barrier is readily overcome if $T>T_{\mathrm{b}}=K / \mathrm{k}_{\mathrm{B}} \ln \left(1 / \omega \tau_{0}\right)$, where $\omega$ is related to the observation time $t=2 \pi / \omega$. In our case, $\omega$ is the sweep frequency of the external magnetic field used to measure $\chi . T_{\mathrm{b}}$ is the blocking temperature, defined by the temperature where the ensemble reaches half of its thermodynamic equilibrium susceptibility $\chi_{\text {eq }}$. For $T>T_{\mathrm{b}}$ the particles are superparamagnetic, and $\chi(T)=\chi_{\mathrm{eq}}(T)$. For $T<T_{\mathrm{b}}$ the particles are blocked in a fixed magnetization state (up or down), and hence $\chi(T)=0$. This is the state where information can be stored. In the vicinity of $T_{\mathrm{b}}$ the system is determined by the kinetics of barrier crossing.

To fully capture the kinetics and thermodynamics of the ensemble of monodisperse particles, we first calculated the zero-field susceptibility by numerically solving the master equations to obtain the occupation of the two energy minima, and by allowing for thermal fluctuations around these minima (see red filled circle data points in Fig. 2). Because we are interested in the limit of small fields, we can also linearize the master equations to describe the kinetics ${ }^{17}$, and multiply with the equilibrium susceptibility $\chi_{\mathrm{eq}}(T)$ to derive the following analytical expression for the real part of the complex zero-field susceptibility, which is the quantity we measure

$$
\chi(T)=\frac{1}{1+\omega^{2} \tau^{2}} \chi_{\mathrm{eq}}(T) .
$$

For $\chi_{\text {eq }}(T)$ (see thin red line in Fig. 2), the analytical solution is given by ${ }^{12}$ :

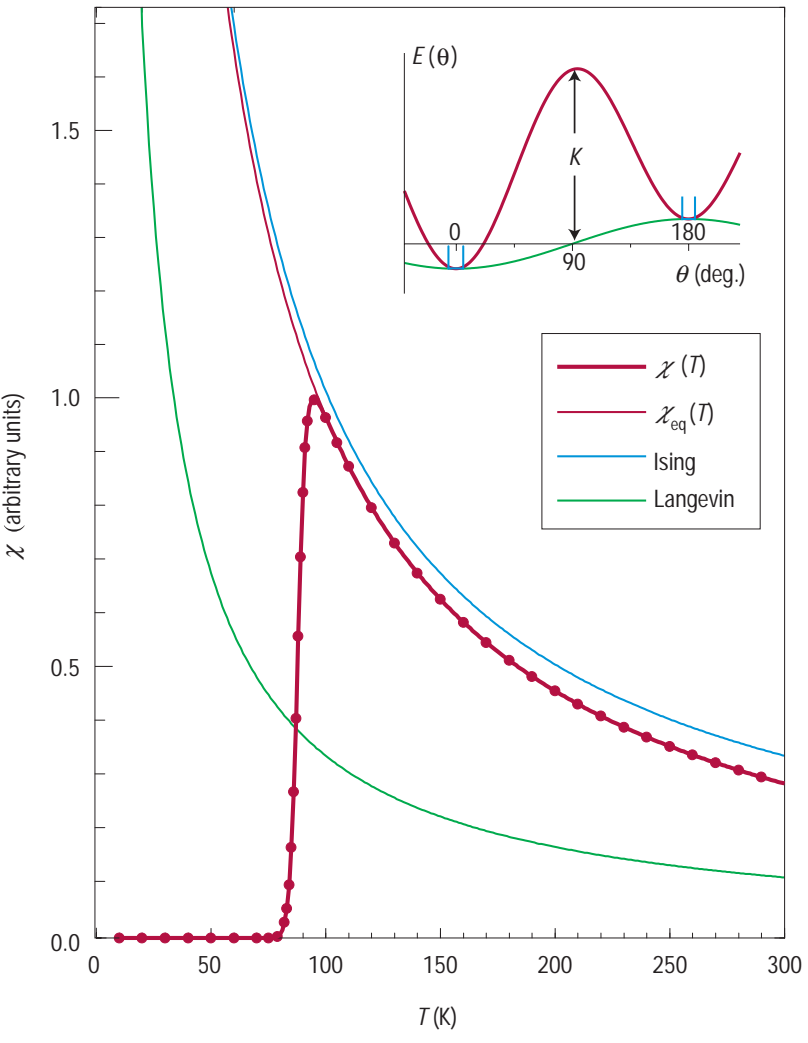

Figure 2 Zero-field magnetic susceptibility $\chi(T)$ for an ensemble of monodisperse, uniaxial particles. Different models are compared for $K=200 \mathrm{meV}$, $M=1,500$ atoms $\times 2.1 \mu_{\mathrm{B}}=182 \mathrm{meVT}^{-1}$, triangular field sweep with $\omega=0.3 \mathrm{~Hz}$. The analytical model $(\chi(T)$, thick red curve) perfectly describes the blocking to superparamagnetic transition as seen by comparison to the numerical calculation (red circles). The equilibrium zero-field susceptibility, $\chi_{\mathrm{eq}}$, is shown as a thin red curve. Inset: Energy of a uniaxial monodomain particle as a function of the orientation of $\boldsymbol{M}$ with respect to an out-of-plane external field $\boldsymbol{H}$ in the Ising model (two states: up and down), in the Langevin model (continuum of states, no anisotropy, Eis given by Zeemann energy $\boldsymbol{H M}$, and in the full model incorporating the anisotropy energy $K . \chi(T)$ derived from the Ising and Langevin models are shown in the main figure for comparison. Curve colours are as for the main figure.

$$
\chi_{\mathrm{eq}}(T)=M^{2}\left[\frac{\exp \left(K / \mathrm{k}_{\mathrm{B}} T\right)}{\sqrt{\pi K \mathrm{k}_{\mathrm{B}} T} \operatorname{Erfi}\left(\sqrt{K / \mathrm{k}_{\mathrm{B}} T}\right)}-\frac{1}{2 K}\right]
$$

with Erfi being the imaginary error function. Figure 2 shows that our analytical solution for $\chi(T)$ (thick red line) perfectly reproduces the numerical one. Note that the numerical model takes into account the full $E(\Theta)$ curve, whereas the analytical one contains only the maximum energy $K$ through the relaxation time. The agreement between the two shows that the small field limit is justified. Despite the fact that the particles are monodisperse, the transition from blocking to superparamagnetic takes place over a finite temperature window of $\Delta T=2 \mathrm{k}_{\mathrm{B}} T_{\mathrm{b}}^{2} / K$. For comparison, we also show the infinite anisotropy limit, leading to a two-state system (Ising model, see blue curves in main figure and inset), and the vanishing anisotropylimit, characterized by an occupation of all orientations of $M$ (Langevin model, green curves). With increasing temperature, $\chi_{\text {eq }}$ goes from one limit to the other and therefore its decay is slightly steeper than the $1 / T$ behaviour 


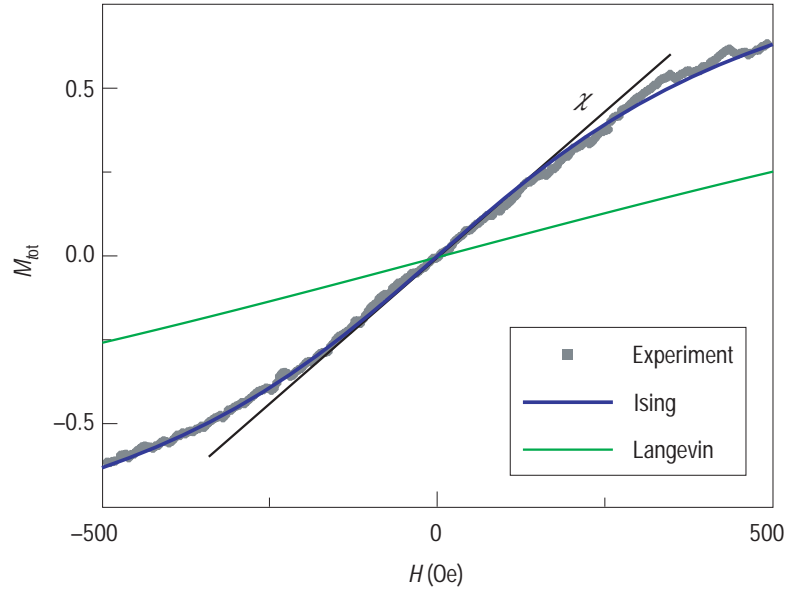

Figure 3 Magnetization $\left(M_{t 0}\right)$ of the sample shown in Fig. $1 \mathrm{~b}$ as a function of out-ofplane field. $T=150 \mathrm{~K}$, triangular field sweep with $\omega=0.6 \mathrm{~Hz}$. $M_{\mathrm{tot}}$ is normalized to the Kerr signal obtained for saturation of a single monolayer film, taking the coverage of 0.4 monolayers into account. The Ising fit is shown as a blue line and yields $m=2.1 \mu_{B}$ when taking the size distribution into account. The green line is the Langevin model with this value. The Langevin model gives equally perfect agreement with the data, but for this an unrealistically high value of $m=6 \mu_{\mathrm{B}}$ has to be assumed.

characterizing the two limiting cases. It is seen that the Ising model is a good approximation for $\chi(T)$ if $T_{\mathrm{b}}<T<2 T_{\mathrm{b}}$, whereas the Langevin model may only be used for very high temperatures. $\chi(T)$ of an ensemble of polydisperse particles is given by summing over the individual particles, each one represented by given values of $M$ and $K$ determined from their area and perimeter using different assumptions of the origin of $M$ and $K$.

For the determination of the magnetic moment $m$ per atom, we used MOKE to measure the normal component of the magnetization vector $M_{\text {tot }}$ of the ensemble of particles, $M_{\mathrm{tot}}$ as a function of applied field at $T>T_{\mathrm{b}}$ for the largest particles of the sample shown in Fig. 1b. The data are displayed as $M_{\mathrm{tot}}(H)$ in Fig. 3 and show the typical superparamagnetic reversible S-shaped curve. The magnetization reaches 0.6 of its saturation value at our experimentally available field of \pm 500 Oe. To reduce the number of parameters, we assume that each atom has the same magnetic moment $m$, thus the moment of an island containing $s$ atoms is given by $M=s m$. This assumption is justified because $m$ varies by much less than $20 \%$ for the size range of interest ${ }^{18}$. As demonstrated above, the Ising model is a good approximation just above $T_{\mathrm{b}}$. Applying this model and taking the size distribution into account leads to the blue curve, perfectly fitting the data and yielding $m=2.1 \pm 0.2 \mu_{\mathrm{B}}$ per atom. This value is in very good agreement with the one expected from taking the sum of the calculated Co spin moment ${ }^{19}$ $m_{\mathrm{Co}, \mathrm{S}}=1.8 \mu_{\mathrm{B}}$, its measured orbital moment ${ }^{20} m_{\mathrm{Co,L}}=0.2 \mu_{\mathrm{B}}$, and the measured polarization of $\mathrm{Pt}$ at its interface with Co amounting to ${ }^{21}$ $m_{\mathrm{Pt}}=0.2 \mu_{\mathrm{B}}$. When the size distribution is unknown, one typically represents the real system by an ensemble of monodisperse particles, all having the average size. We emphasize that this generally yields erroneous results, here it would give $m=3.0 \pm 0.2 \mu_{\mathrm{B}}$ per atom. Moreover, using the Langevin model would yield an unrealistic $m=6 \pm 1 \mu_{\mathrm{B}}$ per atom.

The susceptibility at zero field $\chi$ (the indicated slope in Fig. 3 ) is displayed in Fig. 4a as a function of temperature. It shows a broad transition from blocking to a maximum at $100 \mathrm{~K}$, and subsequently exhibits a roughly $1 / T$ decrease. The full curves are inferred from using the size and perimeter distributions to calculate $\chi(T)$ for the ensemble of a

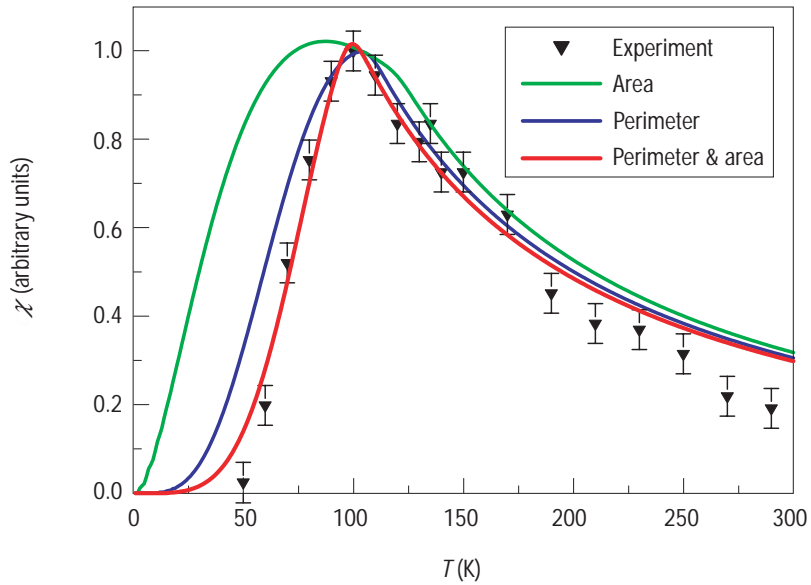

b

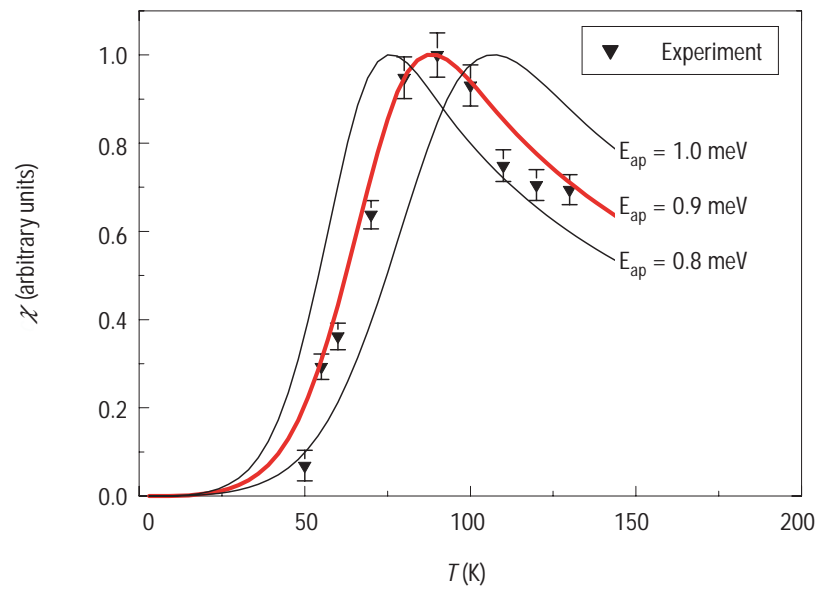

Figure 4 Temperature dependence of the zero-field magnetic susceptibility $\chi(I)$. Measurements by MOKE for compact and ramified islands (triangular field sweep with $\omega=0.6 \mathrm{~Hz}$ and $\pm 0.05 \mathrm{~T}$ amplitude). a, Compact islands: full curves are fits associating the same anisotropy energy $E_{\mathrm{a}}$ to all atoms per island (green), solely to the perimeter atoms (blue), and to both (red). b, Ramified islands with ideal fit $E_{\mathrm{ap}}=0.9 \mathrm{meV}$ (best fitfor $\left.E_{a s}=-0.09 \mathrm{meV}\right)$ and the curves for $0.8\left(E_{\mathrm{as}}=-0.06 \mathrm{meV}\right)$ and $1.0 \mathrm{meV}\left(E_{\mathrm{as}}=-0.12 \mathrm{meV}\right)$ showing the conservative choice of the error margin.

particles from our analytical expression with different assumptions on the origin of the anisotropy energy per island, $K$. First, we assume that each atom contributes the same amount, $K=s E_{\mathrm{a}}$, with $E_{\mathrm{a}}$ denoting the anisotropy per atom (green curve). Second, we assume that only the perimeter atoms contribute to the anisotropy, $K=p E_{\mathrm{a}}$, with $p$ the perimeter length in atoms (blue curve). Finally, we assume that surface atoms contribute the amount $E_{\mathrm{as}}$, and perimeter atoms $E_{\mathrm{ap}}$, thus $K=s E_{\text {as }}+p E_{\text {ap }}$ (red curve). The only free parameter of the fits is the anisotropy $E_{\mathrm{a}}$ attributed to the surface, or to the perimeter atoms, or to both. Because $\chi(T)$ is dominated by terms containing $K / T$, a variation of $E_{\mathrm{a}}$ stretches or compresses the curves along the xaxis, while keeping their origin fixed. However, there is no way to change their overall shape, which is controlled by the size and perimeter distributions.

It is obvious that attributing the anisotropy of the nanostructures entirely to the surface or interface fails because the distribution of the green curve is far too broad. In addition, the inferred value of $E_{\mathrm{a}}=0.047 \pm 0.005 \mathrm{meV}$ per atom is comparable to Co bulk. This small value can only be reconciled with typical thin film values of $0.1 \mathrm{meV}$ per atom when taking the shape anisotropy into account. The latter favours 
Mparallel to the surface, and thus has a negative value. Starting from the saturation magnetization of bulk Co we calculate a demagnetizing energy of $E_{\text {shape }}=-0.090 \mathrm{meV}$ per atom. We note that a variation of the demagnetization factor with island size is expected, however, this variation is small justifying our assumption of a constant value of 1 for all island sizes ${ }^{22}$. With $E_{\mathrm{a}}=E_{\text {cryst }}+E_{\text {shape }}$ this would yield $E_{\text {cryst }}=0.137 \mathrm{meV}$ per atom, which is in reasonable agreement with thin-film values. However, the disagreement of the shape of this fit makes clear that the magnetic properties of nanostructures cannot be rationalized by simply scaling down thin-film properties. The blue curve fits our data much better, and associates $E_{\mathrm{a}}=0.57 \pm 0.05 \mathrm{meV}$ to the perimeter atoms and zero contribution to the surface atoms in the interior of the islands. Finally, the best fit is obtained when we take the shape anisotropy into account and associate $E_{\mathrm{ap}}=0.9 \pm 0.1 \mathrm{meV}$ to the perimeter and $E_{\mathrm{a}}=-(0.03 \pm 0.01) \mathrm{meV}$ to the surface atoms. From the latter value we infer an interface magnetocrystalline energy of $E_{\text {cryst }}=0.06 \pm 0.01 \mathrm{meV}$ per atom, which is more than counterbalanced by $E_{\text {shape }}$. This means that without the anisotropy of the perimeter atoms, the islands would be magnetized in-plane.

The results derived above for compact monolayer islands (Fig. 1b) are fully confirmed for the two other morphologies shown in Fig. 1. The case of the ramified monolayer-high islands (Fig. 1a) is shown in Fig. $4 \mathrm{~b}$.Again, the only way to fit the data is to attribute different $E_{\mathrm{a}}$ values to perimeter and surface atoms. The best fit is obtained for $E_{\mathrm{ap}}=0.9 \pm 0.1 \mathrm{meV}\left(E_{\mathrm{as}}=-(0.09 \pm 0.03) \mathrm{meV}\right)$ confirming the value derived for compact islands and showing its independence of island shape. The theoretical curves for the two extremes of the error margin are far off the data, illustrating that the given error does not result from freedom in fitting, it is caused by uncertainties in the absolute length of the island perimeter due to convolution with the shape of the STM tip. Also, the compact bilayer islands show an anisotropy energy of $E_{\mathrm{a}}=0.9 \pm 0.1 \mathrm{meV}$ per perimeter atom, and the atoms in the interior of the islands favour in-plane magnetization due to the shape anisotropy overwhelming the magnetocrystalline anisotropy. Owing to the dominant role of perimeter atoms, we find that ramified islands have the highest anisotropy for a given size. Altogether, this suggests that the reduced coordination of perimeter atoms generally induces extreme anisotropy energies. Making use of this effect may enable one to reduce the particle size much further than formerly anticipated, without running into the so-called superparamagnetic limit. Our finding also opens new possibilities to separately tune the anisotropy and moment of nanostructures. To exemplify this, and to illustrate once more the role of perimeter atoms, we produced bimetallic, one-monolayer-high islands with a non-magnetic core $(\mathrm{Pt})$ surrounded by a magnetic rim only 2-3 atoms wide (Co), see Fig. 5. We took care that the rim was at least two atoms wide to establish long-range ferromagnetic order-in monatomic Co chains at Pt steps ferromagnetic order has been reported ${ }^{23}$ to exist onlybelow $15 \mathrm{~K}$. It is seen from Fig. $5 \mathrm{~b}$ that keeping the anisotropy energy per perimeter atom fixed to the value inferred above for the pure Co islands perfectly reproduces the switching behaviour. The bimetallic islands have identical anisotropy to their equally shaped pure Co counterparts, however, they have a much smaller overall moment due to their non-magnetic core.

In the above discussion, we tacitly assumed the absence of particle interactions that were possibly mediated by their dipolar stray field. Our data show that such interactions are indeed absent. On the one hand this is inferred from the agreement between experimental and calculated data for $\chi(T)$ in the superparamagnetic regime, where we observe the predicted close to $1 / T$ decrease. Interactions would considerably flatten this decrease ${ }^{24}$. On the other hand, the absence of interactions is also rationalized by comparing the maximum stray field created by all other islands at the location of one island $H_{\text {dipol }}=100$ Oe (Equation (4) of Ref. 25), with its coercive field, $H_{c}(0 \mathrm{~K})=2 \times 10^{4}$ Oe. This comparison is quite favourable, and signifies that much higher densities of non-interacting particles may be realized for out-of-plane

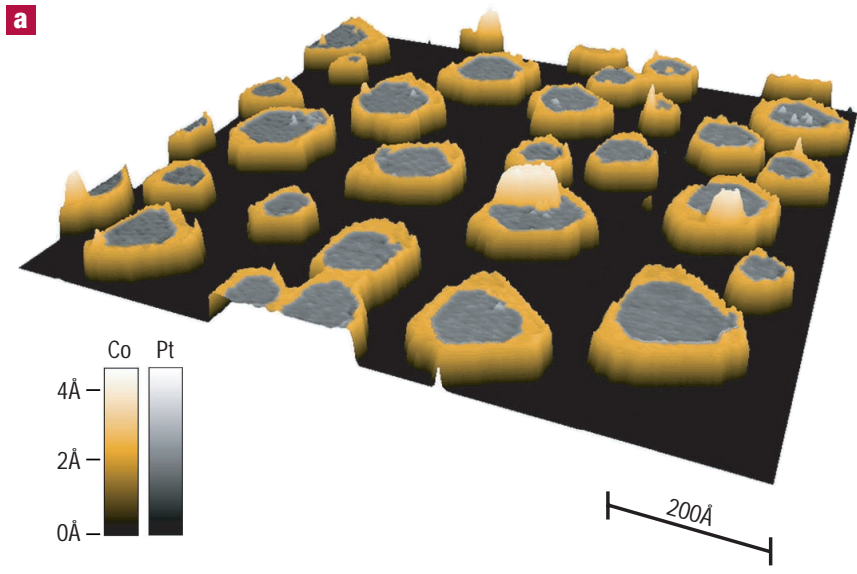

b

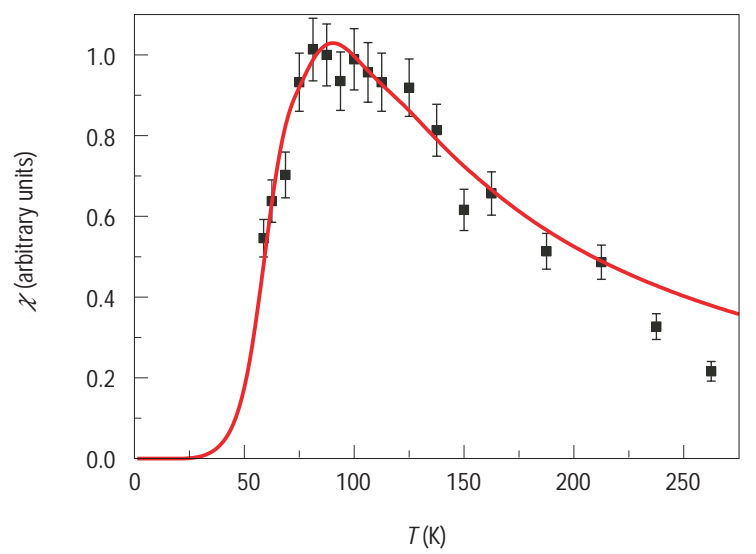

Figure 5 Tailoring magnetic properties in bimetallic islands. a, 3D view of an STM image of one-monolayer-high islands with a Pt core and an approximately 3-atom-wide Co shell (deposition of 0.2 monolayers of Ptat $130 \mathrm{~K}$ and annealing to $760 \mathrm{~K}$ generates the quasi-hexagonal non-magnetic core; subsequent deposition of 0.2 monolayers of $C 0$ at $220 \mathrm{~K}$ creates the Co rim). In the STM topographs, Co can be discerned from Pt by its $0.3 \AA$ larger apparentheight, which was used as the colour code. $\mathbf{b}, \chi(T)$ reveals that the Co-rim/Pt-core islands (square data points) have the same anisotropy as the pure Co islands with equal perimeter length (the red line shows the model with $E_{\mathrm{ap}}=0.9 \mathrm{meV}$ and $E_{\mathrm{as}}=-0.03 \mathrm{meV}$ for $\mathrm{Co}$ ).

magnetization than the density investigated here, which amounts to $2 \times 10^{12}$ particles per square inch.

The central role found for perimeter atoms in the magnetic anisotropy explains several observations reported in literature. The first indication for the important role of step atoms came from a paper ${ }^{26}$ reporting that step decoration with minute amounts of $\mathrm{Cu}$ turns the easy magnetization axis of a Co film 10-20 monolayers thick on $\mathrm{Cu}(100)$ in plane by $90^{\circ}$. This implies that edge atoms are also of crucial importance for the anisotropy of systems with in-plane easy magnetization. X-ray magnetic circular dichroism (XMCD) measurements revealed ${ }^{27}$ a systematic enhancement of the anisotropy energy per atom with reduced size for Co clusters on $\mathrm{Au}(111)$. This finding is perfectly rationalized by our results. The authors of Ref. 27 (see also ref. 28 for the case of Fe/ $\mathrm{Au}(111)$ ), could not attribute $E_{\mathrm{a}}$ values to surface and perimeter atoms, because the only available 
information on the cluster morphology was the average size, deduced from XMCD by applying the Langevin model. Quite generally, the classical approach often used to look for perimeter versus surface effects in epitaxially grown 2D nanostructures has its pitfalls. It consists of investigating a series of coverages and assuming a constant cluster density, and thus that the size is proportional to the coverage. Quantitative results are difficult to derive this way, because the finite width of the size distribution, and the variation of the density due to nucleation and/or coalescence, lead to large systematic errors. This problem is overcome in our method investigating a whole range of sizes at the same time, while explicitly entering the morphology into the analysis. We further note that $E_{\mathrm{a}}$ values of the same magnitude as the ones derived here have recently been reported for Co atoms forming monatomic chains attached to the $\{111\}$-faceted steps of a $\operatorname{Pt}(997)$ surface $^{23}$. Whereas the chains are an ideal test case to investigate magnetism in $1 \mathrm{D}$, the results do not allow extrapolation to the magnetic anisotropy energy of surface versus perimeter atoms. This is due to the different chemical and magnetic environments. Chain atoms attached to Pt steps have two magnetic neighbours, whereas perimeter atoms in nanostructures and thin films have their own species on one side and thus on average four magnetic neighbours. Perimeter atoms are therefore strongly exchange-coupled with each other, establishing ferromagnetic order on a length scale of the island diameter up to $300 \mathrm{~K}$. We find that these atoms have anisotropy energies in the $1 \mathrm{meV}$ range, and that this value is largely independent of the perimeter shape, crystallographic orientation, and also of branch width in the case of the ramified islands.

Combining atomic-scale knowledge of the morphology of an ensemble of polydisperse particles with integral measurements of their magnetic susceptibility enabled us to quantify the contribution of step and surface atoms to the magnetic anisotropy energy of 2D nanostructures. We expect that generally physical properties of 2D nanostructures can be traced back to the constituent atoms when they are measured in conjunction with the morphology. We expect similar advances in the understanding of anisotropy in thin films from the analysis of the number of step and surface atoms. Identifying the role of the differently coordinated atoms brings us a step closer to one of the ultimate goals of materials science, namely, to assemble structures atom by atom in such a way that the ensemble of atoms presents the desired properties.

\section{Received 17 December 2002; accepted 20 May 2003; published 27 J uly 2003.}

\section{References}

1. Chou, S. Y. Patterned magnetic nanostructures and quantized magnetic disks. IEEE Proc. 85, 652-671 (1997).

2. Harris, J. \& Awschalom, D. Thin films squeeze out domains. Phys. World 19-20 (Jan, 1999).

3. Albrecht, M., Rettner, C. T., Moser, A., Best, M. E. \& Terris, B. D. Recording performance of highdensity patterned perpendicular magnetic media. Appl. Phys. Lett. 81, 2875-2877 (2002).
4. Plumer, M. L., Ek, J. v. \& Weller, D. (eds) The Physics of Ultra-High-Density Magnetic Recording (Springer, Berlin, 2001)

5. Woods, S. I., Kirtley, J. R., Sun, S. \& Koch, R. H. Direct investigation of superparamagnetism on Co nanoparticles films. Phys. Rev. Lett. 87, 137205 (2001).

6. Petit, C., Taleb, A. \& Pileni, M. P. Self-organization of magnetic nanosized cobalt particles. Adv. Mater. 10,259-261 (1998).

7. Puntes, V. F., Krishnan, K. M. \& Alivisatos, P. Synthesis, self-assembly, and magnetic behavior of a twodimensional superlattice of single-crystal $\varepsilon$-Co particles. Appl. Phys. Lett. 78, 2187-2189 (2001).

8. Sun, S., Murray, C. B., Weller, D., Folks, L. \& Moser, A. Monodisperse FePt nanoparticles and ferromagnetic FePt nanocrystal superlattices. Science 287, 1989-1992 (2000).

9. Jamet, M. et al. Magnetic anisotropy of a single cobalt nanocluster. Phys. Rev. Lett. 86, 4676-4679 (2001).

10. Held, G. A., Grinstein, G., Doyle, H., Sun, S. \& Murray, C. B. Competing interactions in dispersions of superparamagnetic nanoparticles. Phys. Rev. B 64, 012408 (2001).

11. Black, C. T., Murray, C. B., Sandstrom, R. L. \& Sun, S. Spin-dependent tunneling in self-assembled cobalt-nanocrystal superlattices. Science 290, 1131-1134 (2000).

12. Fruchart, O. et al. Vertical self-organization of epitaxial magnetic nanostructures. J. Magn. Magn. Mater. 239, 224-227 (2002).

13. Brune, H., Giovannini, M., Bromann, K. \& Kern, K. Self-organized growth of nanostructure arrays on strain-relief patterns. Nature 394, 451-453 (1998).

14. Repain, V., Baudot, G., Ellmer, H. \& Rousset, S. Two-dimensional long-range-ordered growth of uniform cobalt nanostructures on a Au(111) vicinal template. Europhys. Lett. 58, 730-736 (2002).

15. Brune, H., Romainczyk, C., Röder, H. \& Kern, K. Mechanism of the transition from fractal to dendritic growth of surface aggregates. Nature 369, 469-471 (1994).

16. Wernsdorfer, W. et al. Experimental evidence of the Néel-Brown model of magnetization reversal. Phys. Rev. Lett. 78, 1791-1794 (1997)

17. Kinzel, W. \& Fischer, K. H. Dynamics of spin glasses. Solid State Commun. 23, 687-690 (1977).

18. Koide, T. et al. Direct Determination of interfacial magnetic moments with a magnetic phase transition in Co nanoclusters on $\mathrm{Au}(111)$. Phys. Rev. Lett. 87, 257201 (2001).

19. Wu, R., Li, C. \& Freeman, A. J. Structural, electronic and magnetic properties of $\mathrm{Co} / \mathrm{Pd}(111)$ and Co/Pt(111). J. Magn. Magn. Mater. 99, 71-80 (1991).

20. Nakajima, N. et al. Perpendicular magnetic anisotropy caused by interfacial hybridization via enhanced orbital moment in Co/Pt multilayers: magnetic circular x-ray dichroism study. Phys. Rev. Lett. 81, 5229-5232 (1998).

21. Ferrer, S. et al. Surface X-ray diffraction from $\mathrm{Co} / \mathrm{Pt}(111)$ ultrathin films and alloys: Structure and magnetism. Phys. Rev. B 56, 9848-9857 (1997).

22. Aharoni, A. Demagnetizing factors for rectangular ferromagnetic prisms. J. Appl. Phys. 83, 3432-3434 (1998)

23. Gambardella, P. et al. Ferromagnetism in one-dimensional monatomic metal chains. Nature 416, 301-304 (2002).

24. Chantrell, R. W., Walmsley, N., Gore, J. \& Maylin, M. Calculations of the susceptibility of interacting superparamagnetic particles. Phys. Rev. B 63, 024410 (2000).

25. Haginoya, C. et al. Magnetic nanoparticle array with perpendicular crystal magnetic anisotropy. J. Appl. Phys. 85, 8327-8331 (1999).

26. Weber, W., Back, C. H., Bischof, A., Pescia, D. \& Allenspach, R. Magnetic switching in cobalt films by adsorption of copper. Nature 374, 788-790 (1995).

27. Dürr, H. A. et al. Spin and orbital magnetization in self-assembled Co clusters on Au(111). Phys. Rev. B59, R701-R704 (1999).

28. Ohresser, P., Brookes, N. B., Padovani, S., Scheurer, F. \& Bulou, H. Magnetism of small Fe clusters on $\mathrm{Au}(111)$ studied by x-ray magnetic circular dichroism. Phys. Rev. B 64, 104429 (2001).

\section{Acknowledgements}

We gratefully acknowledge the contribution of A. Barraud and financial support from Top-Nano-21 and from the Swiss National Science Foundation.

Correspondence and requests for materials should be addressed to H.B.

Competing financial interests

The authors declare that they have no competing financial interests. 